\title{
Posible sangrado del intestino delgado. ¿Es la videocápsula endoscópica la mejor opción?
}

\section{Is the videocapsule endoscopy the best option for diagnosis of possible bleeding from the small intestine?}

José Augusto Urrego, MD, ${ }^{1}$ William Otero Regino, MD, ${ }^{2 *}$ Martín Gómez Zuleta, MD. ${ }^{2}$

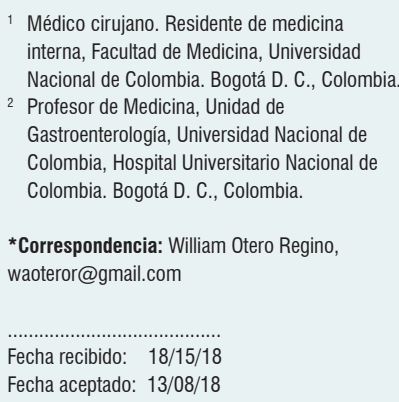

\begin{abstract}
Resumen
En pacientes sin una causa clara de sangrado gastrointestinal luego de una endoscopia digestiva alta y baja, la causa se encontrará en el intestino delgado hasta en el $77 \%$ de los casos. Ante el excelente rendimiento diagnóstico de la videocápsula endoscópica (VCE) para el estudio de este segmento del tracto gastrointestinal, surge la duda de si debería ser el método diagnóstico inicial de este grupo de pacientes con posible sangrado del intestino delgado (PSID) o si la realización de una nueva endoscopia alta y baja o algún método alternativo de estudio del intestino delgado debería serlo. En esta revisión se evalúa y evidencia el rendimiento diagnóstico superior y la mayor seguridad de la VCE como abordaje inicial de pacientes con PSID en relación con otros métodos. Sin embargo, se pone en tela de juicio la mejor costo-efectividad de este abordaje en nuestro medio, que en otros ha sido claramente demostrada.
\end{abstract}

\section{Palabras clave}

Endoscopía del sistema digestivo, endoscopia por cápsula, hemorragia gastrointestinal, intestino delgado.

\begin{abstract}
When neither upper nor lower gastrointestinal endoscopy can find a clear cause of gastrointestinal bleeding, it will eventually be found in the small intestine in up to $77 \%$ of cases. Given the excellent diagnostic performance of video capsule endoscopy for studying this segment of the gastrointestinal tract, the question of whether it should become the initial diagnostic method for patients with possible bleeding from the small intestine arises. The alternatives are to perform additional upper and lower endoscopic procedures or to use some alternative method of studying the small intestine. This review documents and evaluates the superior diagnostic performance and greater safety of videocapsule endoscopy as the initial approach for possible bleeding from the small intestine and compares it with other methods. However, the cost-effectiveness of this approach, clearly demonstrated elsewhere, is questioned in our setting.
\end{abstract}

Keywords

Digestive endoscopy, capsule endoscopy, gastrointestinal hemorrhages, small intestine.

\section{INTRODUCCIÓN}

En el intestino delgado (ID) se producen de $2 \%-10 \%$ de los sangrados gastrointestinales (SGI) (1-3). Sin embargo, cuando la endoscopia digestiva alta (EVDA) y la colonoscopia total (CLNT) no identifican su lugar de origen, en el $77 \%$ de los casos será el ID (4-7).
En el pasado, cuando los exámenes endoscópicos iniciales no encontraban la etiología del sangrado, el cuadro se denominaba sangrado gastrointestinal de origen oscuro (SGIO) (8). A su vez, el SGIO podía ser oculto si su manifestación era anemia ferropénica o se detectaba sangre oculta en las heces, o manifiesto si había manifestaciones clínicas del sangrado como melenas, rectorragia o hema- 
toquecia (8). Actualmente la nomenclatura ha cambiado: cuando los procedimientos endoscópicos son negativos, el cuadro se denomina posible sangrado de intestino delgado (PSID), reservándose el término SGIO para la situación en la cual la EVDA, la CLNT y los exámenes que evalúan el ID son negativos (9).

Las causas más comunes de sangrado de ID en pacientes con PSID son las angiectasias (20\%-55\%), especialmente en ancianos, seguidas por los tumores (10\%-20\%) y la enfermedad de Crohn (EC) (2\%-10\%) (10). Otras causas menos frecuentes incluyen la enfermedad celíaca, divertículo de Meckel, enteropatía por antiinflamatorios no esteroideos (AINE), lesión de Dieulafoy, várices ectópicas, enteropatía hipertensiva portal o enteritis por radiación (10).

La evaluación del ID se puede realizar con diferentes métodos, principalmente enteroscopias, enterorresonancia y videocápsula endoscópica (VCE) (9). Desde su introducción, la VCE ha facilitado la evaluación del ID y en la actualidad se le considera el examen de elección para el estudio de enfermedades de este segmento $(11,12)$. No obstante, varios autores y guías recomiendan repetir la EVDA y la CLNT antes de iniciar el estudio del ID en varios pacientes con PSID $(9,13)$. Este abordaje surge de varios estudios en estos pacientes en quienes con diferentes métodos de evaluación del ID se ha encontrado la causa del sangrado al alcance de la EVDA o de la CLNT en el $2 \%-25 \%$ y $6 \%-23 \%$, respectivamente (14-16). Publicaciones de nuestro medio también recomiendan ese abordaje (17). Sin embargo, ese enfoque ha sido controvertido por varios autores, incluyendo algunos de nuestro país, quienes consideran la VCE como primera línea de evaluación en este grupo de pacientes $(10,18,19)$.

El objetivo de la presente revisión es la descripción y evaluación de la utilidad de la VCE como método de estudio de elección del PSID, después de un primer examen endoscópico alto y bajo negativos, comparándola con el abordaje más tradicional de una segunda endoscopia alta y baja, y también con otros métodos de evaluación del ID.

\section{METODOLOGÍA}

Se realizó una búsqueda bibliográfica en la base de datos PubMed desde los últimos 5 años hasta el 14 de mayo de 2018 utilizando los siguientes términos y metodología "((()(Capsule Endoscopes OR Capsule Endoscope OR Endoscope, Capsule OR Endoscopes, Capsule OR Video Capsule Endoscopes OR Capsule Endoscope, Video OR Capsule Endoscopes, Video OR Endoscope, Video Capsule OR Endoscopes, Video Capsule OR Video Capsule Endoscope)) ) AND ((Endoscopy,
Digestive System OR Digestive System Endoscopies OR Digestive System Endoscopy OR Endoscopies, Digestive System OR Digestive System Endoscopic Surgical Procedures OR Endoscopic, Digestive System, Surgery OR Endoscopic, Digestive System, Surgical Procedure OR Procedure, Digestive System, Endoscopic, Surgical OR Surgical Procedures, Endoscopic, Digestive System OR Procedures, Digestive System, Endoscopic, Surgical OR Procedures, Endoscopic, Digestive System, Surgical OR Surgery, Digestive System Endoscopic OR Surgery, Endoscopic, Digestive System OR Surgical Procedure, Endoscopic, Digestive System OR Digestive System Endoscopic Surgery OR Procedure, Endoscopic, Digestive System, Surgical OR Esophagogastroduodenoscopy OR Esophagogastroduodenoscopies OR Colonoscopy OR Colonoscopies OR Colonoscopic Surgical Procedures OR Colonoscopic Surgical Procedure OR Procedure, Colonoscopic Surgical OR Procedures, Colonoscopic Surgical OR Surgical Procedure, Colonoscopic OR Surgery, Colonoscopic OR Surgical Procedures, Colonoscopic OR Colonoscopic Surgery OR Colonoscopic Surgeries OR Surgeries, Colonoscopic $)))$ AND ((((Intestine, Small OR Intestines, Small OR Small Intestines OR Small Intestine OR Duodenum OR Ileum ORJejunum OR Small Bowel))) AND ((Hemorrhage OR Hemorrhages OR Bleeding $))$ )", especificando la presencia en el título o resumen para cada uno de los términos de la búsqueda, sin límite de fechas, solo en los idiomas español o inglés. Posteriormente, se revisaron los títulos y resúmenes de todos los resultados para identificar los artículos que al concepto de los autores merecieran su revisión total. Adicionalmente, se revisaron algunas referencias de los artículos seleccionados y algunos artículos y guías del conocimiento de los autores.

\section{RESULTADOS}

\section{VCE}

La VCE se concibió hace más de 30 años y, posteriormente, desarrolló de forma simultánea por dos grupos independientes, uno israelí y otro inglés (20-22). Desde entonces se ha convertido en un excelente método de visualización del tracto gastrointestinal, especialmente del ID, donde otros métodos tienen problemas de rendimiento o de facilidades logísticas en su aplicación $(17,23)$. Su refinamiento tecnológico no ha cesado desde su concepción y los nuevos modelos permiten un mejor desempeño y diferentes utilidades (11, $24,25)$. En la actualidad hay varios modelos disponibles para examinar los diferentes segmentos gastrointestinales, de los cuales PillCam es el primero y el más estudiado (GivenImaging; ${ }^{\oplus}$ Yoqneam, Israel) (Tabla 1) (26). 
Tabla 1. VCE disponibles en el mercado (22)

\begin{tabular}{|c|c|c|c|c|c|c|c|c|}
\hline & $\begin{array}{l}\text { PillCam } \\
\text { Colon } 2\end{array}$ & $\begin{array}{c}\text { Pillcam } \\
\text { ESO2 }\end{array}$ & $\begin{array}{c}\text { Pillcam } \\
\text { SB2 }\end{array}$ & $\begin{array}{c}\text { Pillcam } \\
\text { SB3 }\end{array}$ & EndoCapsule & MiroCam & ОМОМ & CapsoVision \\
\hline Longitud, mm & 31 & 26 & 26 & 26 & 26 & 24 & 27,9 & 31 \\
\hline Peso, g & 3,4 & 3,4 & 3,4 & 3 & 3,8 & 3,4 & 6 & - \\
\hline Número de cámaras & 2 & 2 & 1 & 1 & 1 & 1 & 1 & 4 \\
\hline Fotos por segundo & $4-35$ & 14 & 2 & $2-6$ & 2 & 3 & 2 & $12-20$ \\
\hline Sensor de imagen & CMOS & CMOS & CMOS & CMOS & CCD & CCD & CCD & LED \\
\hline Duración de la batería, horas & 10 & 0,5 & 8 & $\geq 11$ & 9 & 11 & 8 & 15 \\
\hline Antenas & 8 & NA & 8 & 8 & 8 & 9 & 14 & Sin transmisión \\
\hline
\end{tabular}

CCD: dispositivo de carga acoplada; CMOS: semiconductor complementario de óxido metálico; ESO: esófago; LED: diodo emisor de luz; SB: small bowel. Modificado de: Gerber J et al. Gastrointest Endosc. 2007;66(6):1188-95.

Con el paso de los años la visualización del ID por la VCE ha venido encontrando aplicaciones en el diagnóstico y seguimiento de diversas patologías como la EC, enfermedad celíaca, tumores del ID y enteropatía inducida por los AINE, entre otras (27-30). Así mismo, este método permite identificar la causa de la anemia ferropénica hasta en el $66 \%$ de los pacientes que no tienen una causa aparente de la misma (31-33). En la última década se ha ampliado su uso, sugiriéndola incluso como una estrategia para la tamización de cáncer colorrectal $(26,34)$, esófago de Barrett (35-37) y várices esofágicas $(38,39)$, entre otras (40). Más recientemente se ha estudiado la posibilidad de utilizarla, adicionalmente, como un instrumento terapéutico en la hemorragia de vías digestivas, como una VCE intraoperatoria en tiempo real (41). Estas utilidades dependerán de que se logren los refinamientos tecnológicos necesarios, cuyo desarrollo es un foco de intensa investigación $(11,25)$.

Por su alta sensibilidad en la evaluación del ID, en 2003 la Food and Drug Administration (FDA) removió su indicación previa como método coadyuvante para el estudio intraluminal del ID, designándolo como primera línea (12). En 2006 el comité de tecnología de la American Society for Gastrointestinal Endoscopy (ASGE) concluyó que la VCE tenía un rendimiento superior a los estudios radiológicos con contraste y a la enteroscopia de empuje (EE) en evaluación de PSID (33). Desde entonces, se ha insistido en que es el método de elección para la visualización del ID después de descartar que la fuente del SGI sea alta o baja (13, 33, 42), siendo incluso considerada el estándar de oro en PSID por algunos $(23,43)$. Como tal, el PSID actualmente es la indicación más frecuente de VCE $(66 \%)(11,44)$. La posibilidad de encontrar la causa del sangrado en el ID en estos pacientes oscila entre $35 \%-77 \%$ (6), dependiendo de si el sangrado es oculto o manifiesto. En una serie de 100 pacientes con PSID se encontró una causa de sangrado en el $12 \%$ de los pacientes con sangrado manifiesto previo, en el $44 \%$ de los pacientes con sangre oculta en las heces y anemia ferropénica, y en el $92 \%$ de los pacientes con sangrado manifiesto actual (45).

\section{Evaluación del ID frente a una segunda endoscopia alta 0 baja}

Cuando un examen endoscópico previo alto y bajo es negativo, la posibilidad de que la fuente sea el ID es mayor a que sea el tracto gastrointestinal superior o inferior (Tabla 2) (10). Por lo tanto, se ha considerado que el ID debería ser el próximo órgano a estudiar, lo que ha sido corroborado por la frecuencia con la que se encuentran causas de sangrado en los pacientes sometidos a VCE en comparación con los que se someten a una segunda EVDA y CLNT. Un estudio australiano encontró que al repetir la EVDA y CLNT solo encontraba una lesión pasada por alto en el $4 \%$ de los pacientes, mientras que la VCE encontró alguna lesión causante probable o posible en por lo menos el $62 \%$ (18).

Por su parte, en casos de PSID se ha encontrado que la EE identifica lesiones que estaban al alcance de la EVDA en el 10 $\%-64 \%$ de los casos $(14,15,46,47)$. Así mismo, con la enteroscopia con doble balón (EDB), se identifican lesiones en el 24\%-25\% que también estaban al alcance de una EVDA (5, 48) y en el $7 \%$ de los casos al alcance de una CLNT (5). Con la VCE ocurre algo similar, al encontrar que en el $2 \%-14 \%$ de los pacientes, las causas estaban al alcance de una EVDA (45, 49-55). No obstante, es necesario tener en cuenta dos 
elementos fundamentales: el primero, que el rendimiento de la VCE y otros métodos de estudio del ID sigue siendo mayor a una segunda endoscopia en casi la totalidad de escenarios $(18,42)$; y el segundo, aunque esas lesiones estaban al alcance de una posible nueva endoscopia, estos estudios no demostraron que las mismas fueran encontradas con la endoscopia, sino con un método alternativo.

Tabla 2. Causas de sangrado en PSID (10)

\begin{tabular}{lc}
\multicolumn{1}{c}{ Causas de PSID } & Porcentaje \\
\hline Lesiones de vías digestivas altas & \\
- Úlceras de Cameron & $5-15$ \\
- Angiectasias & $5-10$ \\
- Várices & $1-5$ \\
- Lesión de Dieulafoy & $2-3$ \\
- Ectasia vascular antral gástrica & $1-2$ \\
- Gastropatía por hipertensión portal & $1-2$ \\
Lesiones del ID & \\
- Angiectasia & \\
- Tumores del ID & $20-55$ \\
- EC & $10-20$ \\
- Enfermedad celíaca & $2-10$ \\
- Divertículo de Meckel & $2-5$ \\
- Enteropatía por AINE & $2-5$ \\
- Lesión de Dieulafoy & 5 \\
- Várices ectópicas & $1-2$ \\
- Enteropatía por hipertensión portal & $1-2$ \\
- Enteritis por radiación & $1-2$ \\
Lesiones digestivas bajas & $<1$ \\
- Angiectasias & \\
- Neoplasias & 2 \\
- Lesión de Dieulafoy & 1 \\
\hline
\end{tabular}

Modificado de: Liu K et al. Aliment Pharmacol Ther. 2011;34(4):416-23.

Dentro de las causas del rendimiento de la VCE se ha propuesto que al no insuflar aire y no distender los segmentos gastrointestinales, este examen sería una endoscopia fisiológica y tendría más posibilidades de identificar lesiones como ectasias vasculares, las cuales la endoscopia convencional no identificaría. Así mismo, para la visualización de muchas de estas lesiones, se necesita perseverancia, dedicación y tiempo. Los endoscopistas atareados frecuentemente no disponen del tiempo suficiente para inspeccionar minuciosamente el estómago, incluida una adecuada retrovisión $y$, en el caso del colon, puede suceder que no se cercioren de que realmente han alcanzado el ciego $(42,51)$.

En 2010 la Sociedad Americana de Endoscopia Gastrointestinal recomendó repetir la EVDA en casos de PSID si la presentación era sospechosa de sangrado alto, repetir la colonoscopia si era sospechosa de sangrado bajo o proceder inmediatamente con la VCE si no se sugería ninguno de los dos (56). Sin embargo, indicó también que en la mayoría de casos la VCE era una primera opción razonable (56). Otros autores han sugerido repetir las endoscopias alta y baja solo si el endoscopista es consciente de no haber realizado una adecuada visualización de las vías digestivas altas o bajas, respectivamente (10).

La Sociedad Europea de Endoscopia Gastrointestinal publicó en 2006 una actualización de sus guías, en la cual sugiere ambiguamente que la VCE es el método de elección en PSID (57). Finalmente, las guías más recientes del Colegio Americano de Gastroenterología (ACG) sobre diagnóstico y manejo de sangrado de ID, publicadas en 2015, recomiendan repetir la EVDA en PSID si el paciente presenta melenas o hematemesis, así como también si en la primera EVDA no se visualizó adecuadamente la mucosa, y repetir la colonoscopia si hay hematoquecia recurrente, sospecha de origen bajo o, nuevamente, si en la primera colonoscopia no hubo adecuada inspección de la mucosa (57). Sin embargo, como lo reconocen los autores de las guías, tales recomendaciones tienen un nivel de evidencia "bajo" o "muy bajo" y están basadas en los hallazgos de causas de PSID que estaban al alcance de los métodos endoscópicos convencionales al usar métodos de estudio del ID $(5,14$, 15, 45-55), lo que comentamos previamente. Así mismo, estas guías sugieren proceder directamente con la VCE si no existen las indicaciones descritas para la segunda endoscopia (9). Sin embargo, la escasez de evidencia se manifiesta al reconocer dentro del texto de estas guías que una EE puede ser considerada en lugar de una segunda EVDA y que la VCE puede utilizarse sin una segunda endoscopia previa para acelerar el abordaje, más aún, teniendo en cuenta la aparente no costo-efectividad del abordaje con una segunda endoscopia (9).

\section{Rendimiento de la videocápsula frente a la EE}

Una publicación de 2005 evaluó el rendimiento de la VCE contra la $\mathrm{EE}$ en 7 estudios prospectivos publicados hasta entonces sobre PSID, encontrando un rendimiento de la VCE de $71 \%$ contra $29 \%$ para la EE (58). Un metaanálisis del mismo año que incluyó 14 estudios encontró un rendimiento del $63 \%$ para la VCE y solo del $28 \%$ para la EE (3). En un segundo metaanálisis con 17 estudios se encontró una diferencia de $37 \%$ a favor de la VCE en PSID cuando se comparó con otros métodos, siendo la EE el principal comparador (59). El Odds ratio (OR) de la VCE para un hallazgo positivo fue 4,3 (intervalo de confianza [IC] $95 \%$ : 3,1-6,0) (59). Más recientemente, un estudio aleatorizado confirmó esos hallazgos con una causa identificada en el $50 \%$ de los casos con VCE y en el $24 \%$ con EE $(p=0,02)(60)$.

Saurin y colaboradores (61) utilizaron como estándar de oro el diagnóstico clínico final, encontrando que la VCE 
tuvo sensibilidad y especificidad de $92 \%$ y $48 \%$, respectivamente, frente al $80 \%$ y $69 \%$ para la EE.

\section{Rendimiento de la videocápsula frente a la enteroscopia con doble balón (EDB)}

Algunos estudios pequeños han comparado directamente la VCE y EDB. Uno de ellos, que incluyó 13 pacientes, encontró que en el PSID el valor diagnóstico fue similar con ambos métodos (62). Un metaanálisis posterior, que incluía 227 pacientes en los que se comparaba el rendimiento de ambos métodos, encontró que la VCE detectaba más causas que la EDB cuando esta última no se hacía combinando el abordaje anal y oral (OR: 1,61; IC 95 \%: 1,07$2,43)$, pero menos cuando la EDB se hacía con tal abordaje (63). Sin embargo, un estudio de ese mismo año concluyó que la VCE más frecuentemente tuvo un diagnóstico etiológico que la EDB por abordaje anal y oral ( $59 \%$ frente al $42 \%)$, aunque la diferencia no fue estadísticamente significativa $(p=0,30)$, probablemente por el pequeño número de pacientes $(n=32)(64)$. En general, el rendimiento de ambos métodos se considera similar, con la ventaja de que la VCE es un método no invasivo y con menos efectos colaterales $(13,65-67)$.

\section{Rendimiento de la videocápsula frente a la enteroscopia intraopertatoria (EIOP)}

La enteroscopia intraoperatoria (EIOP) es considerada por algunos como el mejor método para estudiar completamente el ID y el que tiene el mayor rendimiento etiológico (58 \%-88 \%) (9, 68). Existen pocos estudios que comparan la VCE con la EIOP. Uno de los estudios que se destacan es el de Hartmann y colaboradores (69), en el cual la VCE encontró la causa del sangrado en el $74 \%$ frente al $72 \%$ con la EIOP, sin diferencias estadísticamente significativas. En el metaanálisis mencionado de Triester y colaboradores (3), se encontró un solo estudio que comparara ambos métodos y concluyó un rendimiento similar ( $83 \%$ ), por lo cual la VCE sería el método preferido, ya que no es invasiva y carece de las complicaciones y riesgos de la EIOP $(17,69)$.

\section{Rendimiento de la videocápsula frente a otros métodos}

En algunos estudios pequeños, se ha encontrado que la VCE identifica más frecuentemente causas de sangrado que la enteroclisis por la tomografía axial computarizada (TAC) $(\mathrm{n}=8)(70)$ o por resonancia magnética nuclear $(\mathrm{RMN})(\mathrm{n}=14)(71)$. Por su parte, la enteroclisis convencional tiene un rendimiento de $0 \%-21 \%$ (72-75). En el metaanálisis mencionado previamente se encontraron 3 estudios que comparaban la VCE con enteroclisis con- vencional, encontrando una posible causa en el $67 \%$ de los casos con la primera y en el $8 \%$ con la segunda (3). Así mismo, se ha calculado que la VCE permite modificar el tratamiento en el $66 \%$ de los pacientes con PSID (76), comparado con $10 \%$ al utilizar la enteroclisis convencional (72), con las limitaciones de hacer este tipo de comparaciones en poblaciones diferentes.

También se ha demostrado la superioridad de la VCE comparada con la angiografía estándar, con rendimientos de $53 \%$ y $20 \%$, respectivamente (77). Saperás y colaboradores $(78)$ encontraron que la VCE tenía un rendimiento de $72 \%$, frente al $24 \%$ con angiografía por TAC y $56 \%$ con la angiografía estándar.

\section{Inconvenientes de la videocápsula}

La principal complicación de la VCE es la posibilidad de su retención a lo largo del tracto gastrointestinal (44), la cual puede ocurrir por cicatrices, masas y otras causas de estenosis del lumen, sin una forma adecuada de predecirla con certeza en la actualidad, a excepción de la presencia de signos y síntomas sugestivos de obstrucción intestinal $(17,19)$. La retención de la VCE puede ocurrir en $0 \%$ a $21 \%$ de los pacientes, dependiendo de la población, siendo más frecuente en EC, entre otras $(13,59,80)$. En caso de sospecha clínica de riesgo de retención, se recomienda una enteroTAC o enterorresonancia como métodos imagenológicos para evaluar tal riesgo, o la "cápsula de patencia", la cual identificaría la estenosis, pero al desintegrarse espontáneamente no originaría una emergencia por la obstrucción total del ID $(13,81)$.

Otros posibles eventos adversos están relacionados con dificultades técnicas e incluyen mal funcionamiento de la cápsula por problemas con la batería y problemas con la transferencia de datos $(76,82-84)$. En casos de vaciamiento gástrico demorado, puede permanecer mucho tiempo en el estómago, con consumo innecesario de la batería, la cual se puede consumir, antes de que examine completamente el ID $(82,83)$. Dado que no insufla aire, no tiene la capacidad de distender el ID y, por lo tanto, habrá zonas en las cuales no hay una adecuada exposición de la superficie mucosa y no identificaría lesiones en esos sitios $(13,22)$. Una limitación inherente a la VCE es que en la actualidad sigue siendo un método diagnóstico, sin la posibilidad de tomar biopsias o realizar intervenciones terapéuticas (13). Adicionalmente, tiene contraindicaciones, que incluyen el embarazo (no hay investigaciones en este grupo) y los problemas de deglución por el riesgo de aspiración traqueal, por lo que es necesario avanzarla endoscópicamente en este último caso $(6,26)$.

A pesar de las limitaciones mencionadas, entre todos los métodos de evaluación del ID que pudieran tener un 
rendimiento similar, la VCE es el más seguro. Por ejemplo, la EIOP se ha asociado con múltiples complicaciones, que incluyen lesión de los vasos mesentéricos, íleo prolongado, hematoma, infección, perforación e incluso mortalidad por complicaciones postoperatorias $(17,69)$. Así mismo, la EDB, por su naturaleza invasiva, menor tolerabilidad y necesidad de sedación o anestesia, en la actualidad se recomienda solo cuando la VCE haya encontrado lesiones tratables con la EDB, cuando se requieran biopsias o si luego de una VCE sin hallazgos persiste la sospecha de sangrado de ID (65-67, 85-87).

\section{Costo-efectividad de la videocápsula frente a una nueva endoscopia alta o baja}

Una variable que puede ayudar a decidir entre estas dos estrategias son los costos. Un estudio retrospectivo realizado en Grecia evaluó el rendimiento de la VCE en PSID y concluyó que esta es más costo-efectiva que repetir la EVDA y CLNT (42). Realizar una nueva EVDA y CLNT a todos los pacientes hubiese costado $€ 50,050$ (143 pacientes $\mathrm{x} € 350$ ), lo que habría evitado 9 VCE innecesarias, con un gasto de $€ 80,400$ por las VCE necesarias (134 pacientes $X € 600$ ), para un total de $€ 130,450$ con esta estrategia. La estrategia inversa, con realización de VCE a todos los pacientes, tuvo un costo de $€ 85,800$ (143 pacientes $\mathrm{x} €$ 600 ). Esto representa un ahorro de $€ 312,2$ por paciente. Debe destacarse que estos pacientes habían sido evaluados en el pasado en promedio con 2 EVDA (Rango 1-3) y 2 colonoscopias (rango 1-3), por lo que no puede descartarse que, en caso de menos evaluaciones endoscópicas previas, se hubieran encontrado más causas al alcance de estos métodos tradicionales.

Se podría intentar extrapolar los datos mencionados a los costos locales de estos métodos diagnósticos, conscientes de las claras limitaciones de este tipo de extrapolaciones. Para los cálculos se tendrán en cuenta las tarifas que el Hospital Universitario de la Universidad Nacional le ofrece a las entidades que le remiten sus pacientes: EVDA con sedación: \$ 375 000, CLNT con sedación: \$ 413000 y VCE: \$ 6000 000. En caso de hacerlo, se tendría que el abordaje inicial con una segunda endoscopia alta y baja, seguidas por VCE en casos de no hallazgos, tendría un costo de \$ 916684000 (143X \$ $788000+134$ X \$ 6000000$)$. Por su parte, la realización de VCE directamente a todos los pacientes costaría \$ 858000000 (143 X \$ 6000 000), representando un ahorro de $\$ 410377,6$ por paciente.

En el estudio australiano mencionado previamente se encontró también un costo mayor en la estrategia de repetir la endoscopia alta y baja y, en caso de no hallazgos, proceder a la VCE, que con el uso de la estrategia inversa (el uso de la VCE directamente para todos los pacientes) (18). Con la primera estrategia se evaluaban 50 pacientes con ambas endoscopias ( $\$ 1273$ por paciente) y 47 pacientes con VCE ( $\$ 1801,9$ por paciente); con la segunda se realizaban 50 VCE y, a lo sumo, 26 nuevas endoscopias altas y bajas. Esto llevaba a un gasto total de \$ 148364 al proceder a segunda endoscopia alta y baja, mientras que el gasto era de $\$ 123$ 199 con una VCE inmediata, representando un ahorro de \$503000 por paciente. Si se extrapolaran los hallazgos de este estudio a los costos de nuestra institución se tendría que la estrategia inicial de una nueva EVDA y CLNT costaría \$ 321400000 (50 X \$ $788000+47$ X \$ 6000000$)$ ), mientras que la estrategia inversa, con VCE inicial seguida por nuevas endoscopias en caso de no hallazgos costaría \$ 320488000 (50 X \$ $6000000+26$ X \$ 788000$)$. En este caso se tendría un ahorro de $\$ 18240$ por paciente con la VCE como estrategia inicial.

Un tercer estudio internacional, en este caso italiano, encontró también una mejor costo-efectividad con el uso de VCE en el estudio del PSID al compararla con los demás métodos de evaluación de estos pacientes, incluidos $\mathrm{EE}$, enteroclisis, EVDA, colonoscopia, TAC, angiografía, RMN, ecografía, gammagrafía y EIOP (43). El costo promedio de la VCE para llegar a un diagnóstico fue de $€ 2091$, mientras que el de los otros métodos fue $€ 3829$.

En Colombia no hay estudios que comparen los diferentes métodos para investigar el PSID, mucho menos estudios de costo-efectividad al respecto. Sin embargo, se han publicado dos series de casos retrospectivas que describen el uso de VCE en 97 (19) y 50 pacientes (88) con PSID. En esos estudios, la causa del sangrado fue identificada en 91,7 \% (89/97) y $58 \%(29 / 50)$, respectivamente. En el estudio de Galiano y colaboradores (19), las lesiones encontradas estaban al alcance del endoscopio estándar en el $24,7 \%$ de los pacientes con PSID (24/97): esófago: 1, estómago: 10, duodeno: 8 y colon: 5. Para el caso del estudio de García del Risco y colaboradores (88), 14 \% de los pacientes presentó lesiones al alcance del endoscopio estándar (7/50), distribuidas de la siguiente manera: una en el esófago, dos en el estómago, una en el duodeno y tres en el colon derecho.

$\mathrm{Al}$ realizar un análisis de costo-efectividad con estos datos, si se procediera con una nueva EVDA y CLNT para todos los pacientes del primer estudio (19) se gastarían COP \$ 76436000 en endoscopias (97 X COP \$ 788000 ), lo que evitaría el uso de $24 \mathrm{VCE}$, con un gasto por VCE entonces de $\$ 438000000$ ( $73 \times \$ 6,000,000)$, para un total de \$ 514436000 con esta estrategia. Por el contrario, la realización directa de VCE a todos los pacientes costaría \$ 582000000 (97 X \$ 6000000 ), representando un gasto adicional de \$ 696536 por paciente con el uso inmediato de la VCE. Para el segundo estudio (88), la realización de una nueva EVDA y CLNT, seguidas por VCE en caso de no hallazgo de la causa del PSID, costaría \$ 297400000 
(50X \$ $788000+43$ X \$ 6000000$)$, mientras que la realización directa de la VCE costaría \$300 000000 (50 X $\$ 6000000)$. Esto representa un costo adicional de $\$ 52000$ por paciente si se procede inmediatamente con la VCE. Sin embargo, debe recalcarse que estos estudios no fueron diseñados para análisis de costo-efectividad y que además de esos no existen otros estudios que permitan hacer un adecuado análisis de este tipo a nivel local.

Si se extrapolan los hallazgos internacionales a los costos locales también se encuentra una mejor costo-efectividad con la VCE como estrategia inicial después de los exámenes endoscópicos iniciales negativos. Sin embargo, si los cálculos se realizan teniendo en cuenta los rendimientos de las dos series colombianas publicadas, esta estrategia podría ser menos costo-efectiva. El costo de la VCE en otros países es menos del doble de una nueva EVDA y colonoscopia, escenario en el cual parece evidente la mejor costo-efectividad de la VCE como estrategia inicial de estudio de los pacientes con PSID, lo que se ha manifestado en los tres estudios internacionales que hasta el momento han hecho análisis económicos al respecto $(18,42,43)$. Por el contrario, en nuestro medio la VCE es alrededor de 7,6 veces más costosa que una segunda endoscopia alta y baja, de modo que la relación del costo de la VCE con los estudios endoscópicos es muy superior a lo que sucede internacionalmente. Dada esta alta relación no se puede asegurar la costo-efectividad de la VCE si se evitara una segunda EVDA y CLNT. Por lo anterior, consideramos que, en nuestro medio, se necesitan estudios específicos sobre la costo-efectividad de estos métodos.

\section{CONCLUSIONES}

La VCE es un excelente método de evaluación del ID, reconocido como tal por las principales sociedades científicas a nivel mundial. Su rendimiento en PSID es excelente, e incluso considerado el estándar de oro por algunos. Así mismo, es evidente que su rendimiento es mayor al de una segunda endoscopia alta y baja en estos pacientes, detectando incluso causas al alcance de estos procedimientos endoscópicos iniciales pasadas por alto durante ellos. Ante este rendimiento y ante su seguridad y naturaleza no invasiva en relación con otros, este método parece ser la mejor opción para el estudio del PSID luego de una primera EVDA y colonoscopia a la luz de la literatura científica mundial publicada hasta el momento. Así mismo, la mejor costo-efectividad de la VCE ha sido claramente demostrada en el ámbito internacional. Sin embargo, en nuestro medio la VCE es 7,6 veces más costosa que una segunda EVDA y CLNT, por lo que se generan dudas sobre cuál de las dos estrategias es más costo-efectiva en nuestro país. Esta incertidumbre solo cambiará si los precios de la VCE se hacen más competitivos a nivel local o si se realizan estudios prospectivos locales adecuadamente diseñados. Una recomendación que se desprende de los hallazgos internacionales y locales con la VCE es la necesidad de que las EVDA y las CLNT realizadas en pacientes con sangrado manifiesto u oculto se realicen con el más alto estándar de calidad para evitar pasar por alto las lesiones que han detectado las VCE al alcance de esos procedimientos endoscópicos.

\section{REFERENCIAS}

1. Szold A, Katz LB, Lewis BS. Surgical approach to occult gastrointestinal bleeding. Am J Surg. 1992;163(1):90-2; discussion 2-3.

https://doi.org/10.1016/0002-9610(92)90258-S

2. Longstreth GF. Epidemiology and outcome of patients hospitalized with acute lower gastrointestinal hemorrhage: a population-based study. Am J Gastroenterol. 1997;92(3):419-24.

3. Triester SL, Leighton JA, Leontiadis GI, Fleischer DE, Hara AK, Heigh RI, Shiff AD, Sharma VK. A meta-analysis of the yield of capsule endoscopy compared to other diagnostic modalities in patients with obscure gastrointestinal bleeding. Am J Gastroenterol. 2005;100(11):2407-18. https://doi.org/10.1111/j.1572-0241.2005.00274.x

4. Chong J, Tagle M, Barkin JS, Reiner DK. Small bowel pushtype fiberoptic enteroscopy for patients with occult gas- trointestinal bleeding or suspected small bowel pathology. Am J Gastroenterol. 1994;89(12):2143-6.

5. Tee HP, Kaffes AJ. Non-small-bowel lesions encountered during double-balloon enteroscopy performed for obscure gastrointestinal bleeding. World J Gastroenterol. 2010;16(15):1885-9. https://doi.org/10.3748/wjg.v16.i15.1885

6. ASGE Technology Committee, Wang A, Banerjee S, Barth BA, Bhat YM, Chauhan S, Gottlieb KT, Konda V, Maple JT, Murad F, Pfau PR, Pleskow DK, Siddiqui UD, Tokar JL, Rodriguez SA. Wireless capsule endoscopy. Gastrointest Endosc. 2013;78(6):805-15. https://doi.org/10.1016/j.gie.2013.06.026

7. Ell C, Remke S, May A, Helou L, Henrich R, Mayer G. The first prospective controlled trial comparing wireless capsule endoscopy with push enteroscopy in chronic gastrointesti- 
nal bleeding. Endoscopy. 2002;34(9):685-9.

https://doi.org/10.1055/s-2002-33446

8. American Gastroenterological Association medical position statement: evaluation and management of occult and obscure gastrointestinal bleeding. Gastroenterology. 2000;118(1):197-201. https://doi.org/10.1016/S0016-5085(00)70429-X

9. Gerson LB, Fidler JL, Cave DR, Leighton JA. ACG Clinical Guideline: Diagnosis and Management of Small Bowel Bleeding. Am J Gastroenterol. 2015;110(9):1265-87; quiz 88. https://doi.org/10.1038/ajg.2015.246

10. Liu K, Kaffes AJ. Review article: the diagnosis and investigation of obscure gastrointestinal bleeding. Aliment Pharmacol Ther. 2011;34(4):416-23. https://doi.org/10.1111/j.1365-2036.2011.04744.x

11. Hale MF, Sidhu R, McAlindon ME. Capsule endoscopy: current practice and future directions. World J Gastroenterol. 2014;20(24):7752-9. https://doi.org/10.3748/wjg.v20.i24.7752

12. Gay G, Delvaux M, Rey JF. The role of video capsule endoscopy in the diagnosis of digestive diseases: a review of current possibilities. Endoscopy. 2004;36(10):913-20. https://doi.org/10.1055/s-2004-825868

13. Nakamura T, Terano A. Capsule endoscopy: past, present, and future. J Gastroenterol. 2008;43(2):93-9. https://doi.org/10.1007/s00535-007-2153-6

14. Zaman A, Katon RM. Push enteroscopy for obscure gastrointestinal bleeding yields a high incidence of proximal lesions within reach of a standard endoscope. Gastrointest Endosc. 1998;47(5):372-6. https://doi.org/10.1016/S0016-5107(98)70221-4

15. Descamps C, Schmit A, Van Gossum A. “Missed” upper gastrointestinal tract lesions may explain "occult" bleeding. Endoscopy. 1999;31(6):452-5. https://doi.org/10.1055/s-1999-151

16. Tang SJ, Christodoulou D, Zanati S, Dubcenco E, Petroniene R, Cirocco M, Kandel G, Haber GB, Kortan P, Marcon NE. Wireless capsule endoscopy for obscure gastrointestinal bleeding: a single-centre, one-year experience. Can J Gastroenterol. 2004;18(9):559-65. https: / / doi.org/10.1155/2004/205685

17. Sandoval Riveros C, Lúquez Mindiola A, Marulanda Fernandez H, Otero Regino W. Sangrado del intestino delgado: enfoque y tratamiento Rev Colomb Gastroenterol. 2017;32(3):245-57. https://doi.org/10.22516/25007440.156

18. Gilbert D, O’Malley S, Selby W. Are repeat upper gastrointestinal endoscopy and colonoscopy necessary within six months of capsule endoscopy in patients with obscure gastrointestinal bleeding? J Gastroenterol Hepatol. 2008;23(12):1806-9. https://doi.org/10.1111/j.1440-1746.2008.05643.x

19. Galiano de Sánchez MT, Sánchez Arciniegas F, Pineda Ovalle LF. Experiencia clínica del uso de la videocápsula endoscópica en el diagnóstico de patología del intestino delgado. Rev Col Gastroenterol. 2009;24(1):17-25.
20. Swain P. Wireless capsule endoscopy. Gut. 2003;52 Suppl 4:iv48-50. https://doi.org/10.1136/gut.52.suppl_4.iv48

21. Swain CP, Gong F, Mills TN. Wireless transmission of a color television moving image from the stomach using a miniature CCD camera, light source and microwave transmitter [abstract]. Gut. 1996;39:A26. https://doi.org/10.1016/S0016-5107(97)80063-6

22. Gerber J, Bergwerk A, Fleischer D. A capsule endoscopy guide for the practicing clinician: technology and troubleshooting. Gastrointest Endosc. 2007;66(6):1188-95. https://doi.org/10.1016/j.gie.2007.06.003

23. Mustafa BF, Samaan M, Langmead L, Khasraw M. Small bowel video capsule endoscopy: an overview. Expert Rev Gastroenterol Hepatol. 2013;7(4):323-9. https://doi.org/10.1586/egh.13.20

24. Hartmann D, Eickhoff A, Damian U, Riemann JF. Diagnosis of small-bowel pathology using paired capsule endoscopy with two different devices: a randomized study. Endoscopy. 2007;39(12):1041-5. https://doi.org/10.1055/s-2007-966943

25. Schostek S, Schurr MO. European research on wireless endoscopy--the VECTOR project. Stud Health Technol Inform. 2013;189:193-9.

26. Bouchard S, Ibrahim M, Van Gossum A. Video capsule endoscopy: perspectives of a revolutionary technique. World J Gastroenterol. 2014;20(46):17330-44. https://doi.org/10.3748/wjg.v20.i46.17330

27. Kalla R, McAlindon ME, Drew K, Sidhu R. Clinical utility of capsule endoscopy in patients with Crohn's disease and inflammatory bowel disease unclassified. Eur J Gastroenterol Hepatol. 2013;25(6):706-13. https://doi.org/10.1097/MEG.0b013e32835ddb85

28. Rokkas T, Niv Y. The role of video capsule endoscopy in the diagnosis of celiac disease: a meta-analysis. Eur J Gastroenterol Hepatol. 2012;24(3):303-8. https://doi.org/10.1097/MEG.0b013e32834fa914

29. Cobrin GM, Pittman RH, Lewis BS. Increased diagnostic yield of small bowel tumors with capsule endoscopy. Cancer. 2006;107(1):22-7. https://doi.org/10.1002/cncr.21975

30. Endo H, Hosono K, Inamori M, Kato S, Nozaki Y, Yoneda K, Akiyama T, Fujita K, Takahashi H, Yoneda M, Abe Y, Kirikoshi H, Kobayashi N, Kubota K, Saito S, Matsuhashi $\mathrm{N}$, Nakajima A. Incidence of small bowel injury induced by low-dose aspirin: a crossover study using capsule endoscopy in healthy volunteers. Digestion. 2009;79(1):44-51. https://doi.org/10.1159/000204465

31. Riccioni ME, Urgesi R, Spada C, Cianci R, Pelecca G, Bizzotto A, Costamagna G. Unexplained iron deficiency anaemia: Is it worthwhile to perform capsule endoscopy? Dig Liver Dis. 2010;42(8):560-6. https://doi.org/10.1016/j.dld.2010.01.023

32. Koulaouzidis A, Rondonotti E, Giannakou A, Plevris JN. Diagnostic yield of small-bowel capsule endoscopy in patients with iron-deficiency anemia: a systematic review. 
Gastrointest Endosc. 2012;76(5):983-92.

https://doi.org/10.1016/j.gie.2012.07.035

33. Mishkin DS, Chuttani R, Croffie J, Disario J, Liu J, Shah R, Somogyi L, Tierney W, Song LM, Petersen BT; Technology Assessment Committee, American Society for Gastrointestinal Endoscopy. ASGE Technology Status Evaluation Report: wireless capsule endoscopy. Gastrointest Endosc. 2006;63(4):539-45.

https://doi.org/10.1016/j.gie.2006.01.014

34. Van Gossum A, Devière J. Colon capsule endoscopy: a new tool for colon examination? Discov Med. 2010;9(44):46-50.

35. Sánchez-Yagüe A, Caunedo-Alvarez A, García-Montes JM, Romero-Vázquez J, Pellicer-Bautista FJ, Herrerías-Gutiérrez JM. Esophageal capsule endoscopy in patients refusing conventional endoscopy for the study of suspected esophageal pathology. Eur J Gastroenterol Hepatol. 2006;18(9):977-83. https://doi.org/10.1097/01.meg.0000230094.21911.f8

36. Chavalitdhamrong D, Chen GC, Roth BE, Goltzer O, Sul J, Jutabha R. Esophageal capsule endoscopy for evaluation of patients with chronic gastroesophageal reflux symptoms: findings and its image quality. Dis Esophagus. 2011;24(5):295-8. https://doi.org/10.1111/j.1442-2050.2010.01136.x

37. Romero-Vázquez J, Jiménez-García VA, HerreríasGutiérrez JM. Esophageal capsule endoscopy and Barrett's esophagus: where are we in 2013? Rev Gastroenterol Mex. 2013;78(2):55-6.

https://doi.org/10.1016/j.rgmx.2013.02.002

38. Ishiguro H, Saito S, Imazu H, Aihara H, Kato T, Tajiri H. Esophageal Capsule Endoscopy for Screening Esophageal Varices among Japanese Patients with Liver Cirrhosis. Gastroenterol Res Pract. 2012;2012:946169. https://doi.org/10.1155/2012/946169

39. Lu Y, Gao R, Liao Z, Hu LH, Li ZS. Meta-analysis of capsule endoscopy in patients diagnosed or suspected with esophageal varices. World J Gastroenterol. 2009; 15(10):1254-8. https://doi.org/10.3748/wjg.15.1254

40. Romero-Vázquez J, Argüelles-Arias F, García-Montes JM, Caunedo-Álvarez Á, Pellicer-Bautista FJ, HerreríasGutiérrez JM. Capsule endoscopy in patients refusing conventional endoscopy. World J Gastroenterol. 2014;20(23):7424-33. https://doi.org/10.3748/wjg.v20.i23.7424

41. Yamashita K, Okumura H, Oka Y, Urakami A, Shiotani A, Nakashima H, Matsumoto H, Hirai T, Nakamura M. Minimally invasive surgery using intraoperative real-time capsule endoscopy for small bowel lesions. Surg Endosc. 2013;27(7):2337-41. https://doi.org/10.1007/s00464-012-2777-3

42. Vlachogiannakos J, Papaxoinis K, Viazis N, Kegioglou A, Binas I, Karamanolis D, Ladas S. Bleeding lesions within reach of conventional endoscopy in capsule endoscopy examinations for obscure gastrointestinal bleeding: is repeating endoscopy economically feasible? Dig Dis Sci.
2011;56(6):1763-8.

https://doi.org/10.1007/s10620-011-1592-3

43. Marmo R, Rotondano G, Rondonotti E, de Franchis R, D’Incà R, Vettorato MG, Costamagna G, Riccioni ME, Spada C, D’Angella R, Milazzo G, Faraone A, Rizzetto M, Barbon V, Occhipinti P, Saettone S, Iaquinto G, Rossini FP; Club Italiano Capsula Endoscopica - CICE. Capsule enteroscopy vs. other diagnostic procedures in diagnosing obscure gastrointestinal bleeding: a cost-effectiveness study. Eur J Gastroenterol Hepatol. 2007;19(7):535-42. https://doi.org/10.1097/MEG.0b013e32812144dd

44. Liao Z, Gao R, Xu C, Li ZS. Indications and detection, completion, and retention rates of small-bowel capsule endoscopy: a systematic review. Gastrointest Endosc. 2010;71(2):280-6. https://doi.org/10.1016/j.gie.2009.09.031

45. Pennazio M, Santucci R, Rondonotti E, Abbiati C, Beccari G, Rossini FP, De Franchis R. Outcome of patients with obscure gastrointestinal bleeding after capsule endoscopy: report of 100 consecutive cases. Gastroenterology. 2004;126(3):643-53. https://doi.org/10.1053/j.gastro.2003.11.057

46. Chak A, Cooper GS, Canto MI, Pollack BJ, Sivak MV. Enteroscopy for the initial evaluation of iron deficiency. Gastrointest Endosc. 1998;47(2):144-8. https://doi.org/10.1016/S0016-5107(98)70347-5

47. Landi B, Tkoub M, Gaudric M, Guimbaud R, Cervoni JP, Chaussade S, Couturier D, Barbier JP, Cellier C. Diagnostic yield of push-type enteroscopy in relation to indication. Gut. 1998;42(3):421-5. https://doi.org/10.1136/gut.42.3.421

48. Fry LC, Bellutti M, Neumann H, Malfertheiner P, Mönkemüller K. Incidence of bleeding lesions within reach of conventional upper and lower endoscopes in patients undergoing double-balloon enteroscopy for obscure gastrointestinal bleeding. Aliment Pharmacol Ther. 2009;29(3):342-9. https://doi.org/10.1111/j.1365-2036.2008.03888.x

49. Viazis N, Papaxoinis K, Theodoropoulos I, Sgouros S, Vlachogiannakos J, Pipis P, Markoglou C, Avgerinos A. Impact of capsule endoscopy in obscure small-bowel bleeding: defining strict diagnostic criteria for a favorable outcome. Gastrointest Endosc. 2005;62(5):717-22. https://doi.org/10.1016/j.gie.2005.06.049

50. Estévez E, González-Conde B, Vázquez-Iglesias JL, de Los Angeles Vázquez-Millán M, Pértega S, Alonso PA, Clofent J, Santos E, Ulla JL, Sánchez E. Diagnostic yield and clinical outcomes after capsule endoscopy in 100 consecutive patients with obscure gastrointestinal bleeding. Eur J Gastroenterol Hepatol. 2006;18(8):881-8. https://doi.org/10.1097/00042737-200608000-00014

51. Kitiyakara T, Selby W. Non-small-bowel lesions detected by capsule endoscopy in patients with obscure GI bleeding. Gastrointest Endosc. 2005;62(2):234-8. https://doi.org/10.1016/S0016-5107(05)00292-0 
52. Chong AK, Taylor AC, Miller AM, Desmond PV. Initial experience with capsule endoscopy at a major referral hospital. Med J Aust. 2003;178(11):537-40. https://doi.org/10.5694/j.1326-5377.2003.tb05354.x

53. Sturniolo GC, Di Leo V, Vettorato MG, De Boni M, Lamboglia F, De Bona M, Bellumat A, Martines D, D>Inca R. Small bowel exploration by wireless capsule endoscopy: results from 314 procedures. Am J Med. 2006;119(4):341-7. https://doi.org/10.1016/j.amjmed.2005.08.029

54. Carey EJ, Leighton JA, Heigh RI, Shiff AD, Sharma VK, Post JK, Fleischer DE. A single-center experience of 260 consecutive patients undergoing capsule endoscopy for obscure gastrointestinal bleeding. Am J Gastroenterol. 2007;102(1):89-95. https://doi.org/10.1111/j.1572-0241.2006.00941.x

55. Hoedemaker RA, Westerhof J, Weersma RK, Koornstra JJ. Non-small-bowel abnormalities identified during small bowel capsule endoscopy. World J Gastroenterol. 2014;20(14):4025-9. https://doi.org/10.3748/wjg.v20.i14.4025

56. ASGE Standards of Practice Committee, Fisher L, Lee Krinsky M, Anderson MA, Appalaneni V, Banerjee S, BenMenachem T, Cash BD, Decker GA, Fanelli RD, Friis C, Fukami N, Harrison ME, Ikenberry SO, Jain R, Jue T, Khan K, Maple JT, Strohmeyer L, Sharaf R, Dominitz JA. The role of endoscopy in the management of obscure GI bleeding. Gastrointest Endosc. 2010;72(3):471-9. https://doi.org/10.1016/j.gie.2010.04.032

57. Rey JF, Ladas S, Alhassani A, Kuznetsov K, Committee EG. European Society of Gastrointestinal Endoscopy (ESGE). Video capsule endoscopy: update to guidelines (May 2006). Endoscopy. 2006;38(10):1047-53. https://doi.org/10.1055/s-2006-944874

58. Melmed GY, Lo SK. Capsule endoscopy: practical applications. Clin Gastroenterol Hepatol. 2005;3(5):411-22. https://doi.org/10.1016/S1542-3565(05)00019-4

59. Marmo R, Rotondano G, Piscopo R, Bianco MA, Cipolletta L. Meta-analysis: capsule enteroscopy vs. conventional modalities in diagnosis of small bowel diseases. Aliment Pharmacol Ther. 2005;22(7):595-604. https://doi.org/10.1111/j.1365-2036.2005.02625.x

60. de Leusse A, Vahedi K, Edery J, Tiah D, Fery-Lemonnier E, Cellier C, Bouhnik Y, Jian R. Capsule endoscopy or push enteroscopy for first-line exploration of obscure gastrointestinal bleeding? Gastroenterology. 2007;132(3):855-62; quiz 1164-5. https://doi.org/10.1053/j.gastro.2006.12.002

61. Saurin JC, Delvaux M, Vahedi K, Gaudin JL, Villarejo J, Florent C, Gay G, Ponchon T. Clinical impact of capsule endoscopy compared to push enteroscopy: 1-year followup study. Endoscopy. 2005;37(4):318-23. https://doi.org/10.1055/s-2005-861114

62. Matsumoto T, Esaki M, Moriyama T, Nakamura S, Iida M. Comparison of capsule endoscopy and enteroscopy with the double-balloon method in patients with obscure bleeding and polyposis. Endoscopy. 2005;37(9):827-32. https://doi.org/10.1055/s-2005-870207

63. Chen X, Ran ZH, Tong JL. A meta-analysis of the yield of capsule endoscopy compared to double-balloon enteroscopy in patients with small bowel diseases. World J Gastroenterol. 2007;13(32):4372-8. https://doi.org/10.3748/wjg.v13.i32.4372

64. Nakamura M, Niwa Y, Ohmiya N, Miyahara R, Ohashi A, Itoh A, Hirooka Y, Goto H. Preliminary comparison of capsule endoscopy and double-balloon enteroscopy in patients with suspected small-bowel bleeding. Endoscopy. 2006;38(1):59-66. https://doi.org/10.1055/s-2005-870446

65. Arakawa D, Ohmiya N, Nakamura M, Honda W, Shirai O, Itoh A, Hirooka Y, Niwa Y, Maeda O, Ando T, Goto H. Outcome after enteroscopy for patients with obscure GI bleeding: diagnostic comparison between double-balloon endoscopy and videocapsule endoscopy. Gastrointest Endosc. 2009;69(4):866-74. https://doi.org/10.1016/j.gie.2008.06.008

66. Hadithi M, Heine GD, Jacobs MA, van Bodegraven AA, V Bodegraven AA, Mulder CJ. A prospective study comparing video capsule endoscopy with double-balloon enteroscopy in patients with obscure gastrointestinal bleeding. Am J Gastroenterol. 2006;101(1):52-7. https://doi.org/10.1111/j.1572-0241.2005.00346.x

67. Pasha SF, Leighton JA, Das A, Harrison ME, Decker GA, Fleischer DE, Sharma VK. Double-balloon enteroscopy and capsule endoscopy have comparable diagnostic yield in small-bowel disease: a meta-analysis. Clin Gastroenterol Hepatol. 2008;6(6):671-6. https://doi.org/10.1016/j.cgh.2008.01.005

68. Douard R, Wind P, Panis Y, Marteau P, Bouhnik Y, Cellier $\mathrm{C}$, Cugnenc P, Valleur P. Intraoperative enteroscopy for diagnosis and management of unexplained gastrointestinal bleeding. Am J Surg. 2000;180(3):181-4. https://doi.org/10.1016/S0002-9610(00)00447-5

69. Hartmann D, Schmidt H, Bolz G, Schilling D, Kinzel F, Eickhoff A, Huschner W, Möller K, Jakobs R, Reitzig P, Weickert U, Gellert K, Schultz H, Guenther K, Hollerbuhl H, Schoenleben K, Schulz HJ, Riemann JF. A prospective two-center study comparing wireless capsule endoscopy with intraoperative enteroscopy in patients with obscure GI bleeding. Gastrointest Endosc. 2005;61(7):826-32. https://doi.org/10.1016/S0016-5107(05)00372-X

70. Voderholzer WA, Ortner M, Rogalla P, Beinhölzl J, Lochs $\mathrm{H}$. Diagnostic yield of wireless capsule enteroscopy in comparison with computed tomography enteroclysis. Endoscopy. 2003;35(12):1009-14. https://doi.org/10.1055/s-2003-44583

71. Gölder SK, Schreyer AG, Endlicher E, Feuerbach S, Schölmerich J, Kullmann F, Seitz J, Rogler G, Herfarth H. Comparison of capsule endoscopy and magnetic resonance (MR) enteroclysis in suspected small bowel disease. Int J Colorectal Dis. 2006;21(2):97-104. https://doi.org/10.1007/s00384-005-0755-0 
72. Malik A, Lukaszewski K, Caroline D, Parkman H, DeSipio J, Banson F, Bazir K, Reddy L, Srinivasan R, Fisher R, Miller L. A retrospective review of enteroclysis in patients with obscure gastrointestinal bleeding and chronic abdominal pain of undetermined etiology. Dig Dis Sci. 2005;50(4):649-55. https://doi.org/10.1007/s10620-005-2551-7

73. Kepczyk T, Kadakia SC. Prospective evaluation of gastrointestinal tract in patients with iron-deficiency anemia. Dig Dis Sci. 1995;40(6):1283-9. https://doi.org/10.1007/ BF02065539

74. Moch A, Herlinger H, Kochman ML, Levine MS, Rubesin SE, Laufer I. Enteroclysis in the evaluation of obscure gastrointestinal bleeding. AJR Am J Roentgenol. 1994; $163(6): 1381-4$. https://doi.org/10.2214/ajr.163.6.7992733

75. Rex DK, Lappas JC, Maglinte DD, Malczewski MC, Kopecky KA, Cockerill EM. Enteroclysis in the evaluation of suspected small intestinal bleeding. Gastroenterology. 1989;97(1):58-60. https://doi.org/10.1016/0016-5085(89)91415-7

76. Mylonaki M, Fritscher-Ravens A, Swain P. Wireless capsule endoscopy: a comparison with push enteroscopy in patients with gastroscopy and colonoscopy negative gastrointestinal bleeding. Gut. 2003;52(8):1122-6. https://doi.org/10.1136/gut.52.8.1122

77. Leung WK, Ho SS, Suen BY, Lai LH, Yu S, Ng EK, Ng SM, Chiu PW, Sung JY, Chan FK, Lau JY. Capsule endoscopy or angiography in patients with acute overt obscure gastrointestinal bleeding: a prospective randomized study with long-term follow-up. Am J Gastroenterol. 2012;107(9):1370-6. https://doi.org/10.1038/ajg.2012.212

78. Saperas E, Dot J, Videla S, Alvarez-Castells A, PerezLafuente M, Armengol JR, Malagelada JR. Capsule endoscopy versus computed tomographic or standard angiography for the diagnosis of obscure gastrointestinal bleeding. Am J Gastroenterol. 2007;102(4):731-7. https://doi.org/10.1111/j.1572-0241.2007.01058.x

79. Rana SS, Bhasin DK, Nagi B, Zargar SA, Malhi NS, Sinha SK, Singh K. Comparative evaluation of barium meal follow-through and barium enteroclysis before capsule endoscopy in obscure gastrointestinal bleeding. Hepatogastroenterology. 2012;59(114):418-21. https://doi.org/10.5754/hge10312

80. Sachdev MS, Leighton JA, Fleischer DE, Heigh RI, Hara AK, Post JA, Erickson PJ, Sharma VK. A prospective study of the utility of abdominal radiographs after capsule endoscopy for the diagnosis of capsule retention. Gastrointest Endosc. 2007;66(5):894-900.

https://doi.org/10.1016/j.gie.2007.06.066
81. Enns RA, Hookey L, Armstrong D, Bernstein CN, Heitman SJ, Teshima C, Leontiadis G, Tse F, Sadowski D. Clinical Practice Guidelines for the Use of Video Capsule Endoscopy. Gastroenterology. 2017;152(3):497-514. https://doi.org/10.1053/j.gastro.2016.12.032

82. Saurin JC, Delvaux M, Gaudin JL, Fassler I, Villarejo J, Vahedi K, Bitoun A, Canard JM, Souquet JC, Ponchon T, Florent C, Gay C. Diagnostic value of endoscopic capsule in patients with obscure digestive bleeding: blinded comparison with video push-enteroscopy. Endoscopy. 2003;35(7):576-84. https://doi.org/10.1055/s-2003-40244

83. Mata A, Bordas JM, Feu F, Ginés A, Pellisé M, FernándezEsparrach G, Balaguer F, Piqué JM, Llach J. Wireless capsule endoscopy in patients with obscure gastrointestinal bleeding: a comparative study with push enteroscopy. Aliment Pharmacol Ther. 2004;20(2):189-94. https://doi.org/10.1111/j.1365-2036.2004.02067.x

84. Costamagna G, Shah SK, Riccioni ME, Foschia F, Mutignani M, Perri V, Vecchioli A, Brizi MG, Picciocchi A, Marano P. A prospective trial comparing small bowel radiographs and video capsule endoscopy for suspected small bowel disease. Gastroenterology. 2002;123(4):999-1005. https://doi.org/10.1053/gast.2002.35988

85. Kameda N, Higuchi K, Shiba M, Machida H, Okazaki $\mathrm{H}$, Yamagami H, Tanigawa T, Watanabe K, Watanabe T, Tominaga K, Fujiwara Y, Oshitani N, Arakawa T. A prospective, single-blind trial comparing wireless capsule endoscopy and double-balloon enteroscopy in patients with obscure gastrointestinal bleeding. J Gastroenterol. 2008;43(6):434-40. https://doi.org/10.1007/s00535-008-2182-9

86. Li X, Dai J, Lu H, Gao Y, Chen H, Ge Z. A prospective study on evaluating the diagnostic yield of video capsule endoscopy followed by directed double-balloon enteroscopy in patients with obscure gastrointestinal bleeding. Dig Dis Sci. 2010;55(6):1704-10. https://doi.org/10.1007/s10620-009-0911-4

87. Heine GD, Hadithi M, Groenen MJ, Kuipers EJ, Jacobs MA, Mulder CJ. Double-balloon enteroscopy: indications, diagnostic yield, and complications in a series of 275 patients with suspected small-bowel disease. Endoscopy. 2006;38(1):42-8. https://doi.org/10.1055/s-2005-921188

88. García del Risco F, Arrieta López E. Rendimiento diagnóstico y hallazgos con la cápsula endoscópica en pacientes con sangrado digestivo de origen oscuro en la Clínica Universitaria San Juan de Dios en Cartagena, Colombia. Rev Col Gastroenterol. 2014;29(2):101-11. 